\title{
Identification of exosomal and non-exosomal microRNAs associated with the drug resistance of ovarian cancer
}

\author{
YIWEN FENG $^{1}$, WENZHAO HANG ${ }^{1}$, ZHENYU SANG $^{1}$, SHUANGDI LI $^{1}$, \\ WEI XU ${ }^{1}$, YI MIAO ${ }^{1}$, WEI BAO ${ }^{1}$, XIAOWEI XI $^{1}$ and QIAN HUANG $^{2}$ \\ Departments of ${ }^{1}$ Obstetrics and Gynecology, and ${ }^{2}$ Oncology, \\ Shanghai General Hospital of Nanjing Medical University, Shanghai 200080, P.R. China
}

Received May 10, 2018; Accepted December 10, 2018

DOI: $10.3892 / \mathrm{mmr} .2019 .10008$

\begin{abstract}
MicroRNAs (miRNAs) serve important roles in drug-resistance; however, exosomal miRNAs associated with drug-resistance in ovarian cancer (OC) have not been reported to date. The current study aimed to analyze the drug resistance-associated exosomal miRNAs in original OC cells and their derived exosomes using microarray data downloaded from the Gene Expression Omnibus database (series GSE76449). The chemosensitive OC cell lines SKOV3_ip1, A2780_PAR and HEYA8, as well as the chemoresistant cell lines SKOV3_TR, A2780_CP20 and HEYA8_MDR, were investigated. Differentially expressed miRNAs (DE-miRNAs) were identified using the limma method, and their mRNA targets were predicted using the miRWalk and LinkedOmics database. Functions of target genes were analyzed with DAVID tool, while TCGA data were used to explore the survival association of identified miRNAs. According to the results, 28 DE-miRNAs were found to be common in exosomal and original samples of A2780_CP20 cells, among which the functions of 5 miRNAs were predicted (including miR-146b-5p, miR-509-5p, miR-574-3p, miR-574-5p and miR-760). In addition, 16 and 35 DE-miRNAs were detected for HEYA8_MDR and SKOV3_TR, respectively, with the functions of 4 of these miRNAs predicted for each cell line (HEYA8_MDR: miR-30a-3p, miR-30a-5p, miR-612 and miR-617; SKOV3_TR: miR-193a-5p, miR-423-3p, miR-769-5p and miR-922). It was also reported that miR-183-5p was the only one common miRNA among the three cell lines. Furthermore, miR-574-3p,
\end{abstract}

Correspondence to: Dr Qian Huang, Department of Oncology, Shanghai General Hospital of Nanjing Medical University, 100 Haining Road, Hongkou, Shanghai 200080, P.R. China

E-mail: qianh2011@126.com

Dr Xiaowei Xi, Department of Obstetrics and Gynecology, Shanghai General Hospital of Nanjing Medical University, 100 Haining Road, Hongkou, Shanghai 200080, P.R. China

E-mail: xixiaowei1966@126.com

Key words: ovarian cancer, exosomes, microRNA, drug resistance, cell proliferation
miR-30a-5p and miR-922 may regulate CUL2 to mediate HIF-1 cancer signaling pathway, while miR-183-5p may modulate MECP2, similar to miR-760, miR-30a-5p and miR-922, to influence cell proliferation. Finally, the downregulated miR-612 may promote the expression of TEAD3 via the Hippo signaling pathway, and this miRNA was associated with poor prognosis. In conclusion, the findings of the present study suggested several underlying miRNA targets for improving the chemotherapy sensitivity of OC.

\section{Introduction}

Ovarian cancer (OC) is the leading cause of mortality associated with gynecologic malignancies, with an estimated 52,100 new cases diagnosed and $>22,500$ mortalities in 2015 in China (1). Chemotherapy, such as cisplatin and paclitaxel treatment strategies, is the main therapeutic approach for advanced OC with an International Federation of Gynecology and Obstetrics stage III or IV (2). However, drug resistance significantly limits the long-term effectiveness for cancer patients and thus leads to a poor 5-year survival rate of $\sim 30 \%$ (3). Therefore, understanding the mechanisms contributing to chemotherapy resistance and investigating effective targets to improve resistance remain important challenges that need to be resolved.

MicroRNAs (miRNAs) are small non-coding RNAs with a length of approximately 19-22 nucleotides, which inhibit target mRNA degradation and/or translation by binding to its complementary sequences in the 3'-untranslated region (4). Accumulating evidence has suggested that miRNAs are involved in OC chemoresistance. For instance, Li et al (5) reported that miR-130a was upregulated in the cisplatin-resistant human OC cell line A2780/DDP; thus, the use of miR-130a inhibitors may re-sensitize A2780/DDP cells by inhibiting the expression of multidrug resistance 1 (MDR1) gene. In addition, Zou et al (6) demonstrated a lower expression of miR-429 in SKOV3/DDP cells as compared with that in parental SKOV3 cells, and this downregulated expression of miR-429 was associated with reduced overall survival (OS) and progression-free survival (6). A further study indicated that the zinc finger E-box binding homeobox 1 (ZEB1) may be a direct target of miR-429, and suggested that overexpression of miR-429 may increase the cisplatin sensitivity 
of OC by suppressing ZEB1 expression (6). Furthermore, Zhu et al (7) compared the expression patterns of miRNA genes in paclitaxel-sensitive (SKOV3) and paclitaxel-resistant (SKOV3-TR30) cell lines, and reported that the miR-134 gene cluster was significantly downregulated in SKOV3-TR30 in comparison with SKOV3 cells, while the expression of the miR-17-92 gene cluster was significantly higher in SKOV3-TR30 cells (7). The study by Kim et al (8) demonstrated that treatment with pre-miR-150 significantly induced apoptosis in paclitaxel-resistant SKpac cells, and inhibited cancer cell migration and angiogenesis. The underlying target may be Notch3 downstream proteins (such as NICD3 and HEY2), cell cycle-associated proteins (such as cyclinD3, pS6 and NF- $\kappa$ B) and apoptosis-associated proteins (BCL-2 and BCL-W), which were all significantly downregulated following pre-miR-150 transfection (8). Therefore, targeting specific miRNAs may be a potential approach to improve the chemotherapy sensitivity.

In addition to their intracellular presence, miRNAs can also be enclosed in exosomes and secreted extracellularly to function as mediators of intercellular communication and transfer phenotypic traits of drug-resistant cells into surrounding sensitive cells, facilitating chemotherapy resistance (8). A previous study by $\mathrm{Yu}$ et al (9) reported that the drug-sensitive variant of the MCF-7 breast cancer cell line (namely, MCF-7/S) acquired drug resistance following co-culture with exosomes from the Adriamycin-resistant variant (MCF-7/Adr). The mechanism involved the delivery of miR-222 into MCF-7/S cells by exosomes, and the Adriamycin resistance of MCF-7/S cells was reportedly lost when miR-222 inhibitors were used (9). Qin et al (10) also demonstrated that exosomes of cisplatin-resistant human non-small cell lung cancer cells (A549/DDP) can confer drug resistance to A549 cells, which was exerted in an exosomal miR-100-5p-dependent manner. Furthermore, Wei et al (11) observed that tamoxifen-resistant MCF-7 exosomes entered into tamoxifen-sensitive MCF-7 cells and then released miR-221/222, reducing the expression of target genes P27 and estrogen receptor $\alpha$, and promoting tamoxifen resistance in the recipient cells. Therefore, targeting miRNAs in cancer cells and their exosomes may represent more effective strategies for OC remission.

Although previous studies by the group of Kanlikilicer et al and Rashed et al $(12,13)$ investigated the exosomal miRNAs profiles in three chemosensitive human epithelial OC cell lines and their corresponding chemoresistant cell lines, the analyses reported in these studies did not explore the difference between miRNAs in exosomes and original cells. Thus, to the best of our knowledge, the exosomal miRNAs involved in the chemotherapy resistance of OC have not been reported to date. The aim of the present study was to screen miRNAs associated with chemotherapy resistance in OC cells using the microarray data of Kanlikilicer et al (12) and Rashed et al (13), which may provide underlying targets for improving the chemotherapy sensitivity in clinical practice.

\section{Materials and methods}

Microarray data. An OC chemotherapy-associated miRNA microarray dataset was collected from the Gene Expression Omnibus (GEO) database (http://www.ncbi.nlm.nih. gov/geo/) under accession number GSE76449 (12). This dataset contained original cells, and their derived exosomal samples of 3 chemosensitive human epithelial OC cell lines (including SKOV3_ip1, A2780_PAR and HEYA8) and 3 corresponding chemoresistant cell lines [including SKOV3 TR (Taxol-resistant); A2780_CP20 (cisplatin-resistant) and HEYA8_MDR (multiple drug-resistant)]. Each sample had two biological repeats and, thus, a total of 24 samples were included for analysis. The SKOV3_TR and HEYA8_MDR cell lines were obtained by respectively adding 150 and $300 \mathrm{ng} / \mathrm{ml}$ paclitaxel in RPMI 1640 medium to induce chemotherapy resistance. The A2780_CP20 cell line was developed by sequential exposure of the A2780 cell line to increasing concentrations of cisplatin (13).

Data normalization and identification of differentially expressed miRNAs (DE-miRNAs). The raw data (CEL files) downloaded from the GPL19117 Affymetrix Multispecies miRNA-4 Array platform were preprocessed (including background correction $\log _{2}$ transformation and quantile normalization) with the Robust Multiarray Average function available in the Bioconductor package (http://www.bioconductor.org/packages/release/bioc/html/limma.html).

DE-miRNAs between chemosensitive and chemoresistant cells were identified using the limma method (also known as Linear Models for Microarray data) (14) in the Bioconductor R package (version 3.4.1; http://www.r-project.org/). Student's t-test was used to calculate the P-value. miRNAs were identified as differentially expressed if they exhibited a P-value of $<0.05$ and $\mid \log$ of fold change $(\log F C) \mid$ of $>1$. A Venn diagram was constructed to illustrate the overlap between different cell types, and between exosomes and their cells of origin using the web-based tool (http://bioinformatics.psb. ugent.be/webtools/Venn/).

Target gene prediction. In order to identify the underlying target genes of DE-miRNAs, the miRWalk2.0 database (zmf.umm.uni-heidelberg.de/apps/zmf/mirwalk2/) (15). This database contained a total of 12 bioinformatic algorithms, including DIANA-microT (version 4.0), DIANA-microT-CDS (version 5.0), miRanda-rel (version 1.0), mirBridge (version 1.0), miRDB (version 4.0), miRmap (version 1.0), miRNAMap (version 1.0), PicTar (version 2.0), PITA (version 6.0), RNA22 (version 2.0), RNAhybrid (version 2.1) and Targetscan (version 6.2). Subsequently, the miRNA-target gene interaction network was established and visualized using the Cytoscape software (version 2.8; www.cytoscape. org) (16). Furthermore, LinkedOmics database (www. linkedomics.org) was also used to investigate the negative association between miRNAs and mRNAs in OC samples with Pearson's correlation analysis (17), with $\mathrm{P}<0.01$ set as the threshold value.

Function enrichment analysis. The Database for Annotation, Visualization and Integrated Discovery (DAVID) online tool (version 6.8; http://david.abcc.ncifcrf.gov) (18) was used for Gene Ontology (GO) term and Kyoto Encyclopedia of Genes and Genomes (KEGG) pathway enrichment analyses of all target genes of DE-miRNAs. A P-value of $<0.05$ was set as the cut-off value. 
A

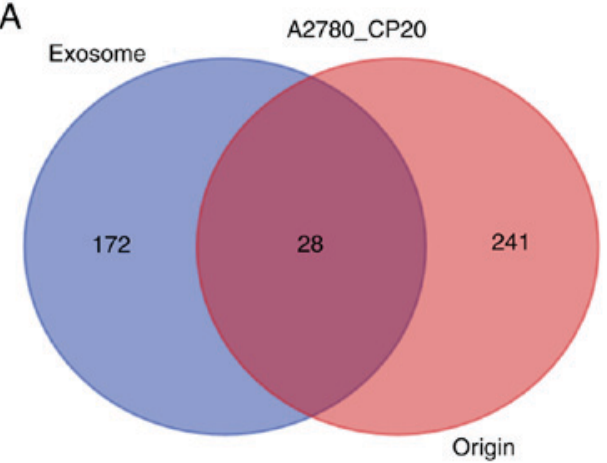

C

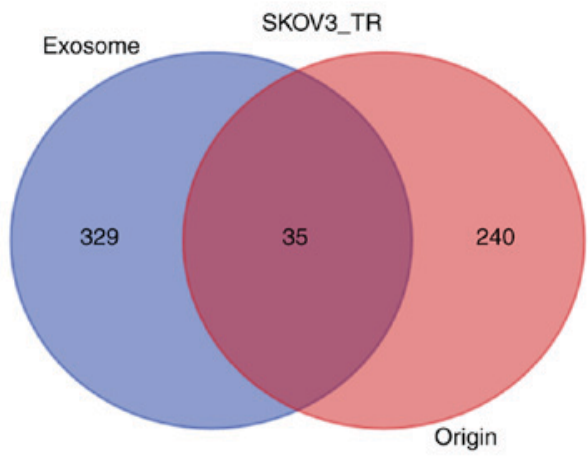

B

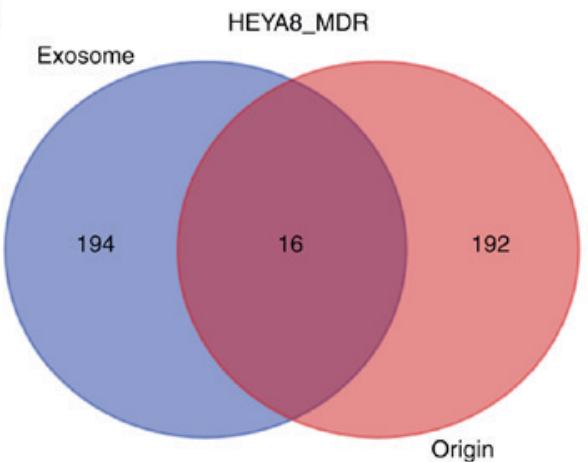

D

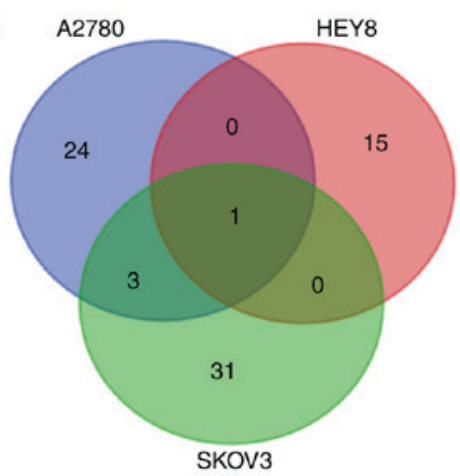

Figure 1. Venn diagrams were used to analyze the common DE-miRNAs screened in three ovarian cancer cells and their exosomes. Chemoresistant and chemosensitive cells were compared, including: (A) A2780_CP20 vs. A2780_PAR, (B) HEYA8_MDR vs. HEYA8, and (C) SKOV3_TR vs. SKOV3_ip1, respectively. (D) Common DE-miRNAs among the three cell lines. DE-miRNAs, differentially expressed microRNAs.

Validation of the correlation between crucial miRNAs and survival. The miRNA Seq data of OC (Level 3) was obtained from The Cancer Genome Atlas (TCGA; https://tcga-data. nci.nih.gov/) to confirm the potential correlation between crucial miRNAs and survival [OS and disease-free survival (DFS)]. The expression levels of crucial miRNAs in OC were analyzed using the FPKM values (referring to the fragments per kilobase per million of mapped reads) from the TCGA data. To identify miRNAs correlated with survival, samples were divided into the low and high expression groups, corresponding to miRNAs with expression levels smaller or greater than the median, respectively. The Kaplan-Meier method with the log-rank test was applied using the GraphPad Prism software (version 5; GraphPad Software Inc., San Diego, CA, USA) to assess the differences in DFS and OS between the high and low expression groups. $\mathrm{P}<0.05$ was considered to denote differences that were statistically significant.

\section{Results}

Identification of DE-miRNAs between chemosensitive and chemoresistant cells. For the exosomal samples, 200 DE-miRNAs were identified between the A2780_CP20 and A2780_PAR cell lines, according to the threshold of $\mathrm{P}<0.05$ and $\mid \log \mathrm{FCl}>1$, including 130 upregulated and 70 downregulated miRNAs. In addition, 210 DE-miRNAs were identified between HEYA8_MDR and HEYA8 cell lines, including 105 upregulated and 105 downregulated miRNAs. A total of 364 DE-miRNAs were identified between the SKOV3_TR and SKOV3_ip1 cell lines, including 147 upregulated and 217 downregulated miRNAs.

For the original cell samples, a total of 269 DE-miRNAs were identified between the A2780_CP20 and A2780_PAR cell lines, including 184 upregulated and 85 downregulated miRNAs. In total, 208 DE-miRNAs were identified between HEYA8_MDR and HEYA8, including 88 upregulated and 120 downregulated miRNAs, while 275 DE-miRNAs were identified between SKOV3_TR and SKOV3_ip1 cell lines, including 150 upregulated and 125 downregulated miRNA.

The Venn diagram analysis (Fig. 1) identified 28 DE-miRNAs that were common in exosomal and original samples between the A2780_CP20 and A2780_PAR cell lines. A total of 16 shared miRNAs were obtained for exosomal and original samples between the HEYA8_MDR and HEYA8 cell lines, while 35 common miRNAs were screened for exosomal and original samples between SKOV3_TR and SKOV3_ip1 (Table I). hsa-miR-183-5p was considered to be particularly important for chemoresistance in an exosomal and non-exosomal manner, since it was the only common miRNA among the three cell lines. However, its expression was upregulated in A2780_CP20 and SKOV3_TR, whereas it was downregulated in HEYA8_MDR cells.

Target genes for DE-miRNAs between chemosensitive and chemoresistant cells. Using the miRWalk2.0 database, a total of 1,056 target genes of 5 common DE-miRNAs in exosomal and original samples between A2780_CP20 and A2780_PAR cell lines were obtained, including miR-146b-5p, miR-509-5p, miR-574-3p, miR-574-5p and miR-760, which 
Table I. Shared differentially expressed miRNA in exosomal and original cells between chemosensitive and chemoresistant cells.

A, A2780_PAR vs. A2780_CP20

\begin{tabular}{|c|c|c|c|c|}
\hline \multirow[b]{2}{*}{ miRNA } & \multicolumn{2}{|c|}{ Exosomes } & \multicolumn{2}{|c|}{ Original cells } \\
\hline & $\log \mathrm{FC}$ & P-value & $\log \mathrm{FC}$ & P-value \\
\hline hsa-miR-212-3p & 5.91 & $6.93 \times 10^{-4}$ & 3.93 & $1.06 \times 10^{-5}$ \\
\hline hsa-miR-574-5p & 5.78 & $3.28 \times 10^{-3}$ & 1.22 & $2.21 \times 10^{-2}$ \\
\hline hsa-miR-320e & 5.25 & $1.91 \times 10^{-2}$ & 1.13 & $2.04 \times 10^{-2}$ \\
\hline hsa-miR-200c-3p & 5.13 & $6.89 \times 10^{-4}$ & 3.8 & $3.58 \times 10^{-5}$ \\
\hline hsa-miR-132-3p & 5.10 & $1.60 \times 10^{-2}$ & 2.83 & $9.69 \times 10^{-5}$ \\
\hline hsa-miR-320d & 4.75 & $3.86 \times 10^{-2}$ & 1.11 & $2.10 \times 10^{-2}$ \\
\hline hsa-miR-27b-3p & 4.68 & $4.40 \times 10^{-2}$ & 1.35 & $8.83 \times 10^{-3}$ \\
\hline hsa-miR-4429 & 4.66 & $1.53 \times 10^{-2}$ & 1.13 & $2.02 \times 10^{-2}$ \\
\hline hsa-miR-183-5p & 4.57 & $1.28 \times 10^{-2}$ & 1.17 & $1.59 \times 10^{-2}$ \\
\hline hsa-miR-185-5p & 4.48 & $1.09 \times 10^{-2}$ & 1.02 & $2.86 \times 10^{-2}$ \\
\hline hsa-miR-23b-3p & 4.41 & $4.26 \times 10^{-2}$ & 1.08 & $2.26 \times 10^{-2}$ \\
\hline hsa-miR-574-3p & 4.39 & $9.11 \times 10^{-3}$ & 1.32 & $1.26 \times 10^{-2}$ \\
\hline hsa-let-7b-5p & 3.63 & $3.83 \times 10^{-2}$ & 2.07 & $1.81 \times 10^{-3}$ \\
\hline hsa-miR-1972 & 3.47 & $5.52 \times 10^{-3}$ & 1.98 & $1.06 \times 10^{-3}$ \\
\hline hsa-miR-146b-5p & 3.29 & $4.13 \times 10^{-3}$ & 2.43 & $7.15 \times 10^{-4}$ \\
\hline hsa-miR-203a & 2.84 & $6.30 \times 10^{-3}$ & 3.88 & $1.89 \times 10^{-5}$ \\
\hline hsa-let-7g-5p & 2.75 & $7.18 \times 10^{-3}$ & 2.17 & $2.20 \times 10^{-3}$ \\
\hline hsa-miR-760 & 2.18 & $2.95 \times 10^{-2}$ & 1.17 & $2.75 \times 10^{-2}$ \\
\hline hsa-miR-509-5p & 1.98 & $3.05 \times 10^{-2}$ & 1.29 & $9.85 \times 10^{-3}$ \\
\hline hsa-let-7d-5p & 1.93 & $4.83 \times 10^{-2}$ & 1.81 & $1.63 \times 10^{-3}$ \\
\hline hsa-miR-3177-3p & 1.88 & $3.28 \times 10^{-2}$ & 1.87 & $5.20 \times 10^{-3}$ \\
\hline hsa-miR-873-3p & 1.83 & $3.49 \times 10^{-2}$ & 1.95 & $1.81 \times 10^{-3}$ \\
\hline hsa-miR-6872-5p & 1.82 & $4.57 \times 10^{-2}$ & 1.2 & $1.95 \times 10^{-2}$ \\
\hline hsa-miR-3926 & 1.81 & $4.73 \times 10^{-2}$ & -1.24 & $3.04 \times 10^{-2}$ \\
\hline hsa-miR-4768-3p & 1.69 & $4.55 \times 10^{-2}$ & 1.16 & $2.31 \times 10^{-2}$ \\
\hline hsa-let-7i-5p & 1.68 & $4.51 \times 10^{-2}$ & 2.14 & $1.10 \times 10^{-3}$ \\
\hline hsa-miR-99a-5p & -2.49 & $1.59 \times 10^{-2}$ & -2.75 & $2.18 \times 10^{-4}$ \\
\hline hsa-miR-19a-3p & -2.29 & $1.89 \times 10^{-2}$ & -1.37 & $7.18 \times 10^{-3}$ \\
\hline
\end{tabular}

B, HEYA8 vs. HEYA8_MDR

\begin{tabular}{lcccc}
\hline & \multicolumn{2}{c}{ Exosomes } & \multicolumn{1}{c}{ Original cells } \\
\cline { 2 - 5 } miRNA & logFC & P-value & logFC \\
\hline hsa-miR-30a-5p & 2.26 & $2.06 \times 10^{-3}$ & 1.18 & 1.12 \\
hsa-miR-6886-5p & 2.03 & $7.62 \times 10^{-3}$ & 1.35 & $2.80 \times 10^{-2}$ \\
hsa-miR-7161-5p & 1.99 & $7.04 \times 10^{-3}$ & 1.06 & $2.60 \times 10^{-2}$ \\
hsa-miR-30a-3p & 1.74 & $1.63 \times 10^{-2}$ & 1.21 & $3.50 \times 10^{-2}$ \\
hsa-miR-3157-5p & 1.63 & $2.57 \times 10^{-2}$ & 1.98 & $1.50 \times 10^{-2}$ \\
hsa-miR-4293 & 1.59 & $3.99 \times 10^{-2}$ & -1.51 & $1.19 \times 10^{-3}$ \\
hsa-miR-598-3p & 1.56 & $3.21 \times 10^{-2}$ & -2.15 & $4.03 \times 10^{-2}$ \\
hsa-miR-4513 & 1.52 & $3.56 \times 10^{-2}$ & -1.17 & $1.06 \times 10^{-2}$ \\
hsa-miR-183-5p & -3.29 & $4.23 \times 10^{-2}$ & -1.43 & $1.10 \times 10^{-3}$ \\
hsa-miR-4436b-5p & -2.80 & $1.91 \times 10^{-4}$ & -1.30 & $1.76 \times 10^{-2}$ \\
hsa-miR-612 & -1.85 & $1.07 \times 10^{-2}$ & 1.75 & $2.25 \times 10^{-2}$ \\
hsa-miR-4533 & -1.83 & $1.18 \times 10^{-2}$ & -1.02
\end{tabular}


Table I. Continued.

\begin{tabular}{|c|c|c|c|c|}
\hline \multirow[b]{2}{*}{ miRNA } & \multicolumn{2}{|c|}{ Exosomes } & \multicolumn{2}{|c|}{ Original cells } \\
\hline & $\operatorname{logFC}$ & P-value & $\log \mathrm{FC}$ & P-value \\
\hline hsa-miR-617 & -1.62 & $2.56 \times 10^{-2}$ & 1.41 & $6.71 \times 10^{-3}$ \\
\hline hsa-miR-503-5p & -1.61 & $2.60 \times 10^{-2}$ & -1.23 & $1.69 \times 10^{-2}$ \\
\hline
\end{tabular}

C, SKOV3_ip1 vs.SKOV3_TR

\begin{tabular}{|c|c|c|c|c|}
\hline \multirow[b]{2}{*}{ miRNA } & \multicolumn{2}{|c|}{ Exosomes } & \multicolumn{2}{|c|}{ Original cells } \\
\hline & $\log \mathrm{FC}$ & P-value & $\log \mathrm{FC}$ & P-value \\
\hline hsa-miR-6716-3p & 9.83 & $7.69 \times 10^{-09}$ & 2.16 & $6.22 \times 10^{-3}$ \\
\hline hsa-miR-4730 & 6.88 & $3.14 \times 10^{-6}$ & 2.37 & $7.10 \times 10^{-4}$ \\
\hline hsa-mir-365a & 4.02 & $1.61 \times 10^{-3}$ & 1.06 & $3.60 \times 10^{-2}$ \\
\hline hsa-miR-25-3p & 2.79 & $2.74 \times 10^{-3}$ & 2.55 & $5.88 \times 10^{-5}$ \\
\hline hsa-miR-99a-5p & 2.12 & $4.11 \times 10^{-3}$ & 6.59 & $2.01 \times 10^{-9}$ \\
\hline hsa-mir-147a & 2.11 & $7.12 \times 10^{-3}$ & 1.08 & $2.85 \times 10^{-2}$ \\
\hline hsa-miR-203a & 2.04 & $4.67 \times 10^{-3}$ & 2.05 & $3.92 \times 10^{-4}$ \\
\hline hsa-miR-132-3p & 1.85 & $4.45 \times 10^{-2}$ & 1.38 & $6.84 \times 10^{-3}$ \\
\hline hsa-miR-128-3p & 1.71 & $1.34 \times 10^{-2}$ & 1.93 & $6.90 \times 10^{-4}$ \\
\hline hsa-miR-8070 & 1.59 & $1.81 \times 10^{-2}$ & -1.37 & $7.70 \times 10^{-3}$ \\
\hline hsa-mir-6894 & 1.58 & $2.56 \times 10^{-2}$ & -1.02 & $3.45 \times 10^{-2}$ \\
\hline hsa-miR-769-5p & 1.55 & $2.05 \times 10^{-2}$ & 1.45 & $5.41 \times 10^{-3}$ \\
\hline hsa-mir-5696 & 1.42 & $3.43 \times 10^{-2}$ & 1.22 & $2.08 \times 10^{-2}$ \\
\hline hsa-miR-423-3p & 1.39 & $3.53 \times 10^{-2}$ & 1.31 & $9.44 \times 10^{-3}$ \\
\hline hsa-miR-183-5p & 1.39 & $3.38 \times 10^{-2}$ & 2.07 & $3.57 \times 10^{-4}$ \\
\hline hsa-miR-922 & 1.30 & $4.85 \times 10^{-2}$ & -1.28 & $3.62 \times 10^{-2}$ \\
\hline hsa-mir-489 & 1.30 & $4.63 \times 10^{-2}$ & 1.02 & $3.32 \times 10^{-2}$ \\
\hline hsa-miR-193b-3p & -6.46 & $8.77 \times 10^{-7}$ & -4.95 & $1.03 \times 10^{-7}$ \\
\hline hsa-miR-31-5p & -3.86 & $1.26 \times 10^{-4}$ & -1.14 & $1.99 \times 10^{-2}$ \\
\hline hsa-miR-146a-5p & -3.22 & $2.19 \times 10^{-4}$ & -4.46 & $1.68 \times 10^{-6}$ \\
\hline hsa-miR-1909-3p & -3.09 & $6.03 \times 10^{-4}$ & -1.42 & $6.10 \times 10^{-3}$ \\
\hline hsa-miR-210-3p & -2.39 & $1.46 \times 10^{-2}$ & -2.32 & $1.51 \times 10^{-4}$ \\
\hline hsa-miR-4315 & -2.3 & $3.36 \times 10^{-3}$ & -1.1 & $2.32 \times 10^{-2}$ \\
\hline hsa-miR-7641 & -2.23 & $7.21 \times 10^{-3}$ & -1.19 & $2.14 \times 10^{-2}$ \\
\hline hsa-miR-193b-5p & -2.12 & $4.62 \times 10^{-3}$ & -2.91 & $7.49 \times 10^{-5}$ \\
\hline hsa-miR-3141 & -1.94 & $6.34 \times 10^{-3}$ & 1.07 & $3.37 \times 10^{-2}$ \\
\hline hsa-miR-202-3p & -1.92 & $8.21 \times 10^{-3}$ & 1.27 & $1.91 \times 10^{-2}$ \\
\hline hsa-miR-3189-3p & -1.63 & $1.70 \times 10^{-2}$ & -1.24 & $1.30 \times 10^{-2}$ \\
\hline hsa-miR-3937 & -1.59 & $2.13 \times 10^{-2}$ & 1.63 & $2.25 \times 10^{-3}$ \\
\hline hsa-miR-1273h-5p & -1.53 & $2.31 \times 10^{-2}$ & -1.05 & $2.95 \times 10^{-2}$ \\
\hline hsa-miR-345-5p & -1.48 & $2.58 \times 10^{-2}$ & -1.05 & $3.01 \times 10^{-2}$ \\
\hline hsa-miR-5196-5p & -1.45 & $2.78 \times 10^{-2}$ & 1.42 & $2.10 \times 10^{-2}$ \\
\hline hsa-miR-193a-5p & -1.40 & $4.60 \times 10^{-2}$ & -1.48 & $4.96 \times 10^{-3}$ \\
\hline hsa-miR-181a-2-3p & -1.36 & $4.24 \times 10^{-2}$ & -2.29 & $1.54 \times 10^{-4}$ \\
\hline hsa-miR-100-5p & -4.29 & $9.87 \times 10^{-5}$ & -3.81 & $9.66 \times 10^{-7}$ \\
\hline
\end{tabular}

FC, fold change; TR, Taxol-resistant; CP, cisplatin resistant; MDR, multiple drug-resistant.

were all upregulated (Fig. 2). Between HEYA8_MDR and HEYA 8 cell lines, a total of 837 target genes of 4 common DE-miRNAs were screened in exosomal and original samples, including the upregulated miR-30a-3p and miR-30a-5p, the downregulated miR-612, as well as miR-617 that was downregulated in exosomal samples, but upregulated in cells 


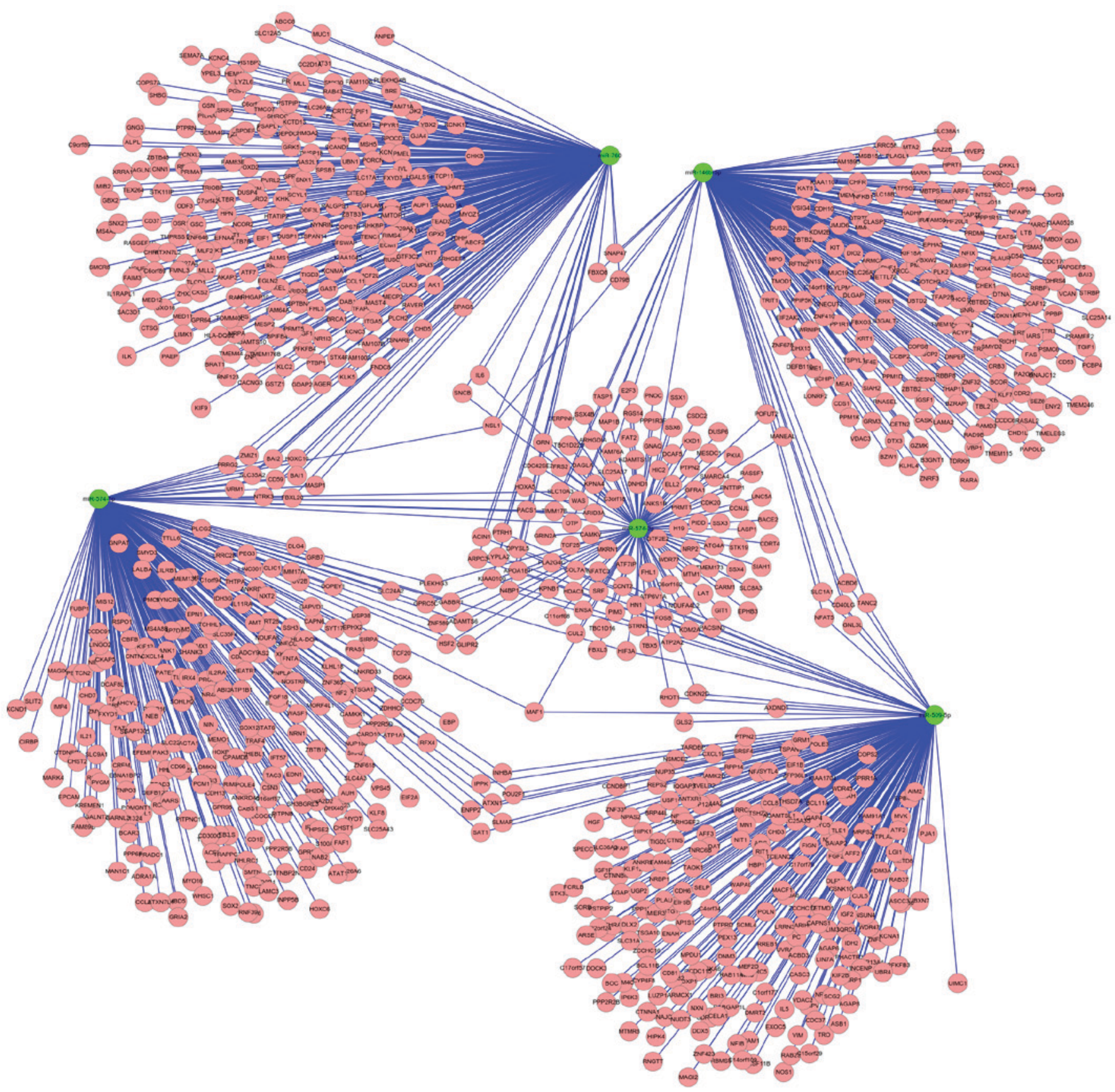

Figure 2. Regulatory network of common differentially expressed miRNAs in ovarian cancer cells and their exosomes, along with their target genes in A2780_CP20 chemoresistant cells. Green circles represent the miRNAs, while red circles represent the target genes. miRNA, microRNA.

(Fig. 3). Between SKOV3_TR and SKOV3_ip1, a total of 773 target genes of 4 common DE-miRNAs were identified in exosomal and original samples, including the upregulated miR-193a-5p, miR-423-3p and miR-769-5p, as well as miR-922 that was upregulated in exosomal samples and downregulated in cells (Fig. 4). Among all these target genes, 18 were found to be common for DE-miRNAs in all three cell lines, including calponin 1 (CNN1), tetratricopeptide repeat, ankyrin repeat and coiled-coil containing 2 (TANC2), F-box and leucine rich repeat protein 20 (FBXL20), glutamate metabotropic receptor 1 (GRM1), ADAM metallopeptidase with thrombospondin type 1 motif 6 (ADAMTS6), cAMP responsive element modulator (CREM), lactalbumin alpha (LALBA), inositol-pentakisphosphate 2-kinase (IPPK), pleckstrin homology and RhoGEF domain containing G4B (PLEKHG4B), shroom family member 4 (SHROOM4), OTU deubiquitinase, ubiquitin aldehyde binding 1 (OTUB1), four and a half LIM domains 3 (FHL3), methyl-CpG binding protein 2 (MECP2), lysine methyltransferase 2A (MLL), TEA domain transcription factor 3 (TEAD3), neurotrophic receptor tyrosine kinase 3 (NTRK3), cullin 2 (CUL2) and HemK methyltransferase family member 1 (HEMK1). No target genes were obtained for hsa-miR-183-5p; thus, LinkedOmics database was subsequently used to screen the genes that were negatively associated with miR-183-5p in order to indirectly predict the role of hsa-miR-183-5p. According to the criterion of $\mathrm{P}<0.01,955$ target genes were obtained. Among them, MECP2 was common with the 


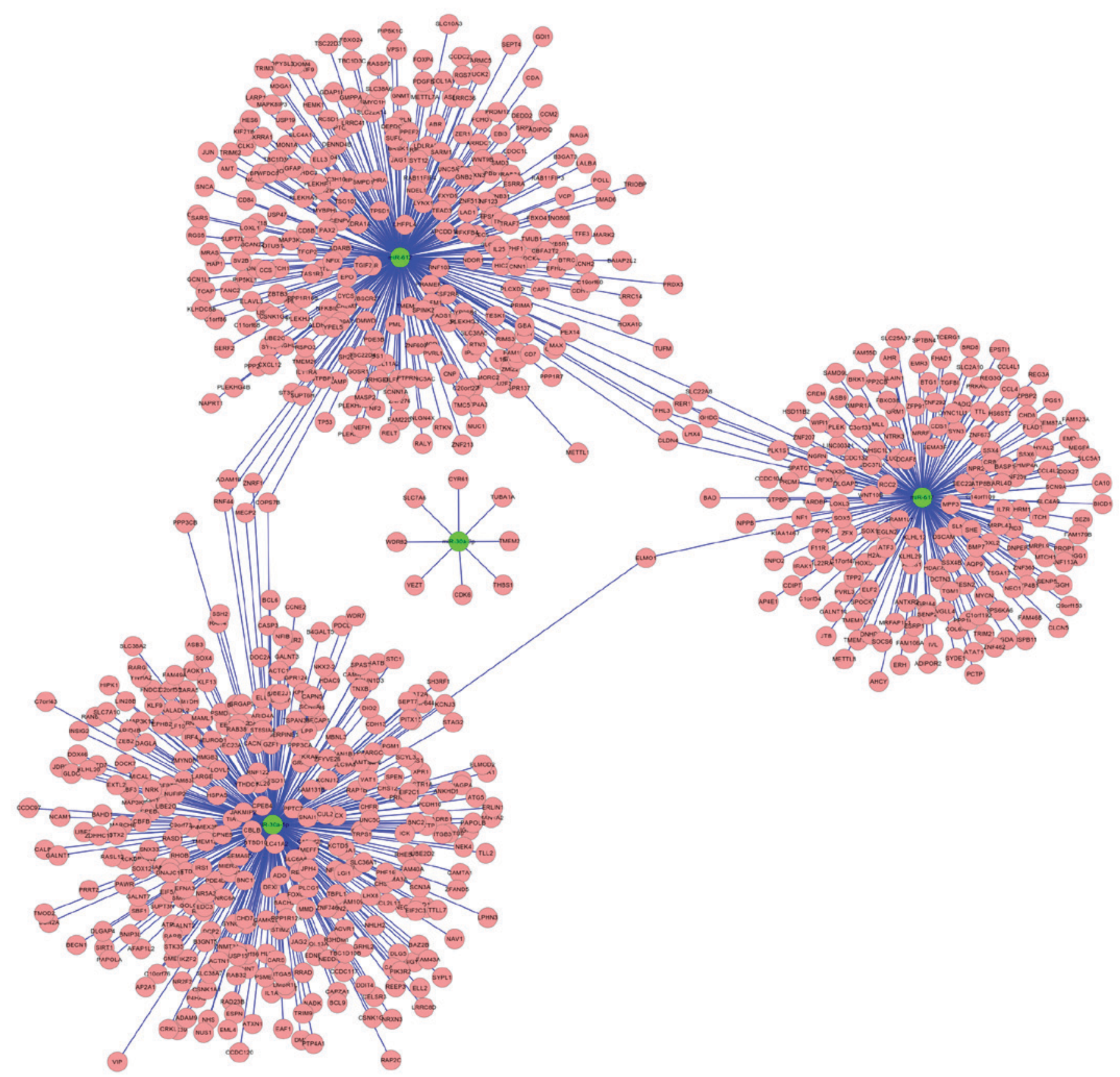

Figure 3. Regulatory network of common differentially expressed miRNAs in ovarian cancer cells and their exosomes, along with their target genes in HEYA8_MDR chemoresistant cells. Green circles represent the miRNAs, while red circles represent the target genes. miRNA, microRNA.

target genes of the aforementioned miRNAs identified by miRWalk2.0 database (Fig. 5), which showed the negative expression relationship between hsa-miR-183-5p and MECP2 in OC samples.

Functional enrichment analysis of common DE-miRNAs in exosomes and their original cells. All target genes of shared DE-miRNAs in exosomes and their original cells were subjected to functional enrichment analysis using DAVID tool. The results identified that $14 \mathrm{KEGG}$ pathways were enriched for A2780_CP20, including glutamatergic synapse (miR-509-5p-GRM1), the hypoxia-inducible factor 1 (HIF-1) signaling pathway (miR-574-3p-CUL2), adrenergic signaling in cardiomyocytes (miR-574-5p-CREM) and the calcium signaling pathway (miR-509-5p-GRM1). In total, 28 KEGG pathways were enriched for HEYA8_MDR, including the neurotrophin signaling pathway (miR-617-NTRK3), pathways in cancer (miR-30a-5p-CUL2), long-term potentiation (miR-617-GRM1), adrenergic signaling in cardiomyocytes (miR-617-CREM) and the Hippo signaling pathway (miR-612-TEAD3). Similarly, 21 KEGG pathways were enriched for SKOV3_TR, including the neurotrophin signaling pathway (miR-922-NTRK3) and the HIF-1 signaling pathway (miR-922-CUL2) (Table II). Based on these results, CUL2 was the commonly enriched target gene in three cell lines.

Furthermore, GO terms were also enriched for the target genes of shared DE-miRNAs in exosomes and their original 


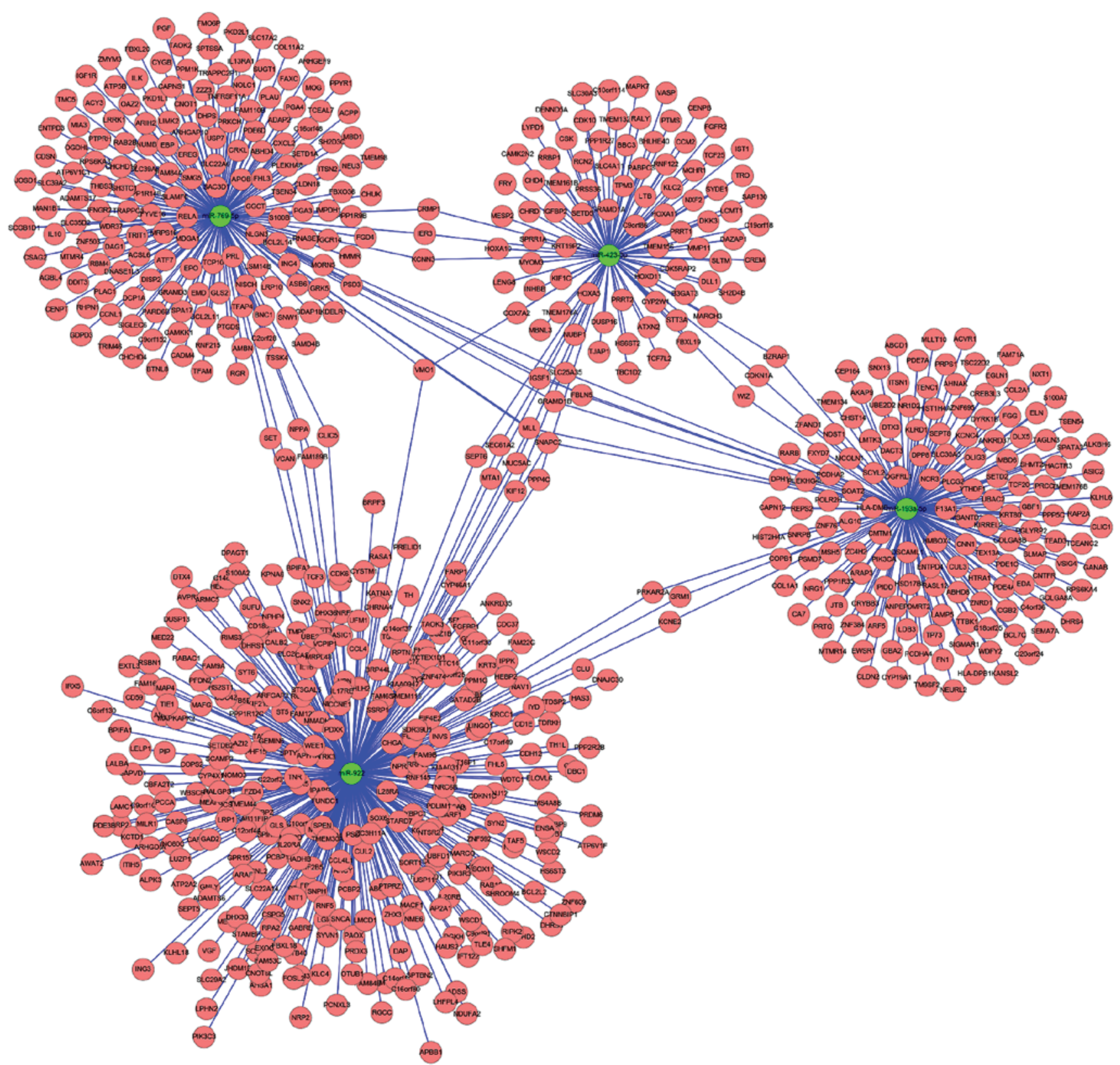

Figure 4. Regulatory network of common differentially expressed miRNAs in ovarian cancer cells and their exosomes, along with their target genes in SKOV3_TR chemoresistant cells. Green circles represent the miRNAs, while red circles represent the target genes. miRNA, microRNA.

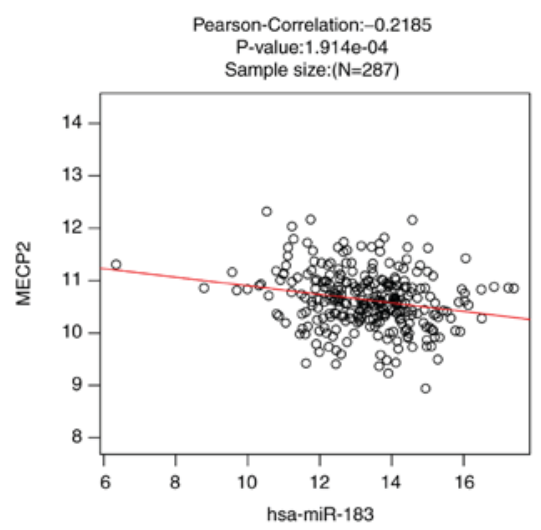

Figure 5. Negative correlation between miR-183-5p and MECP2. miR, microRNA; MECP2, methyl-CpG binding protein 2. This relationship was tested by the Pearson's correlation analysis using the miRNA and RNA seq data of 287 ovarian cancer samples in LinkedOmics database. cells. The results shown in Table III indicated that shared DE-miRNAs may be involved in A2780_CP20 cell chemotherapy resistance by participating in the activation of MAPK activity (miR-509-5p-GRM1), negative regulation of cell proliferation (miR-574-3p-CUL2), G1/S phase transition of the mitotic cell cycle (miR-574-3p-CUL2), response to hypoxia (miR-760-MECP2), and transcription, DNA-templated (miR-574-5p-TEAD3). The analysis also indicated that common DE-miRNAs may be involved in HEYA8_MDR cell chemotherapy resistance by participating in the positive regulation of the apoptotic process (miR-617-NTRK3), negative regulation of cell proliferation (miR-30a-5p-CUL2), negative regulation of transcription, DNA-templated (miR-30a-5p-MECP2), response to hypoxia (miR-30a-5p-MECP2) and positive regulation of transcription from RNA polymerase II promoter (miR-612-TEAD3). Finally, it was observed that common 


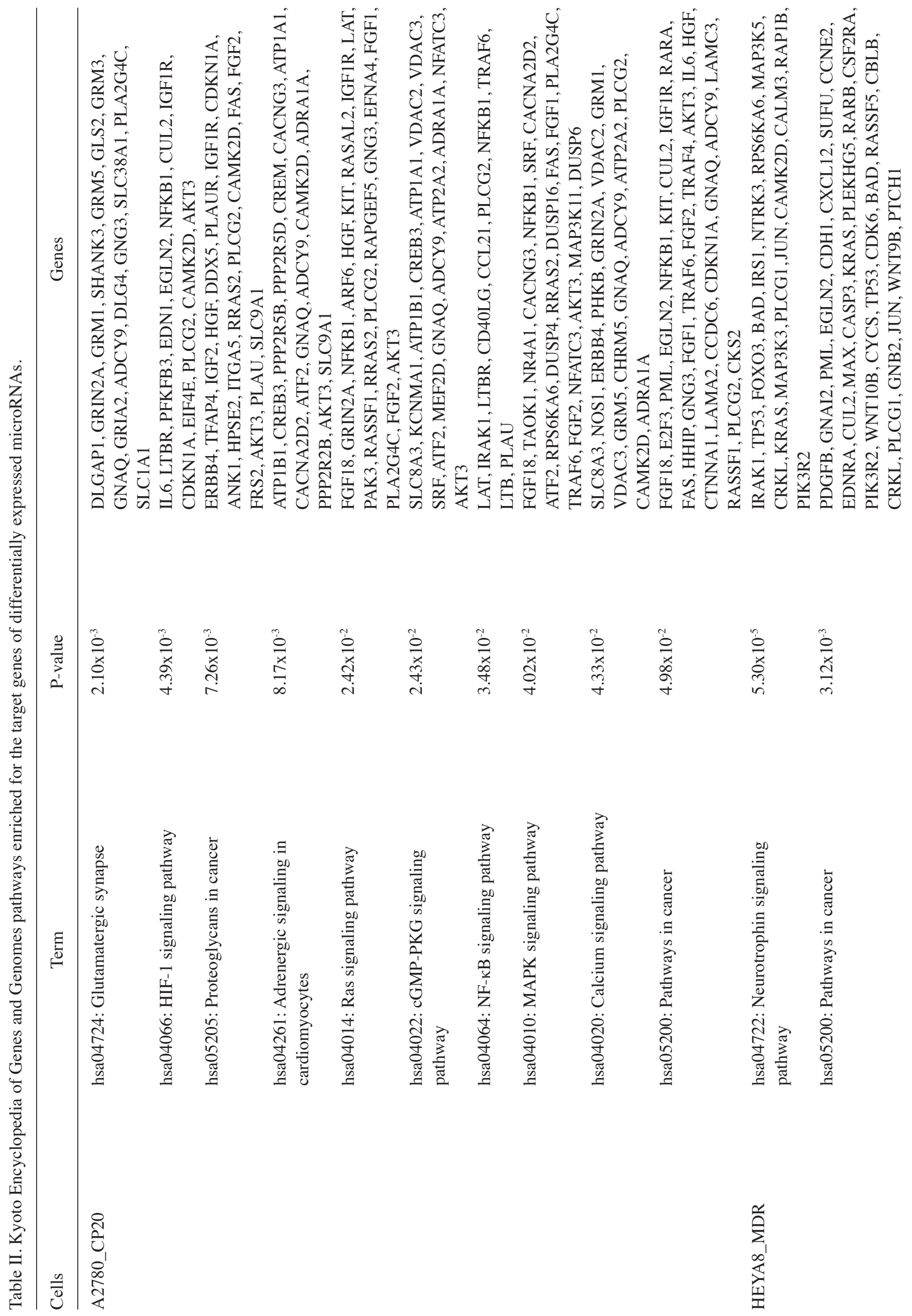


艺光光色

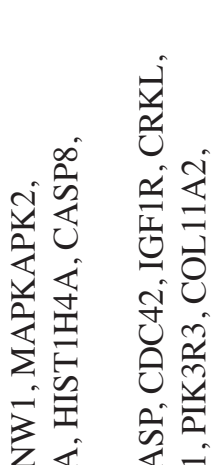

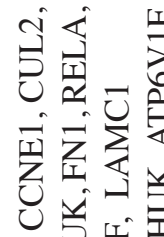

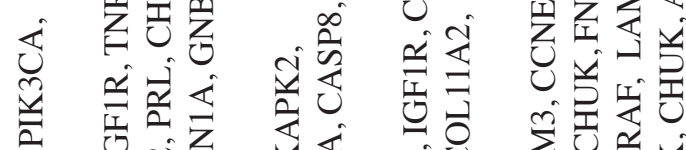

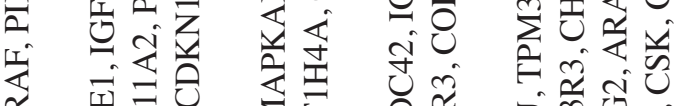

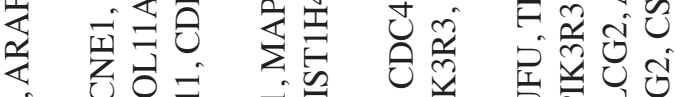
\&

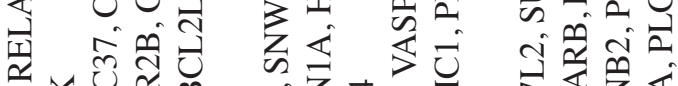

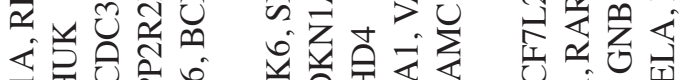

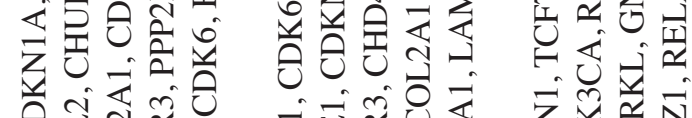

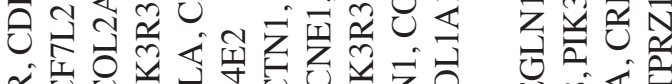

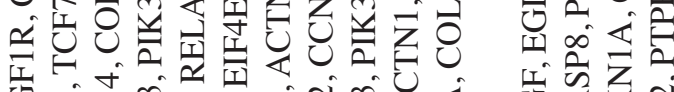

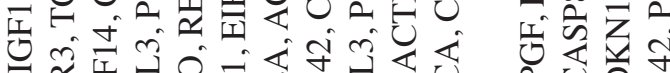

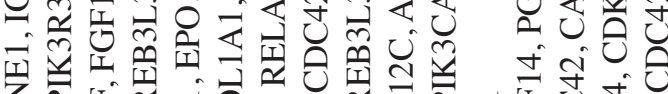

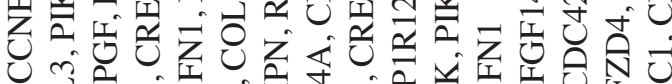

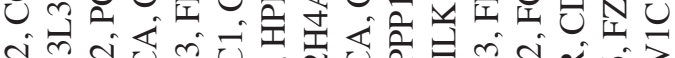

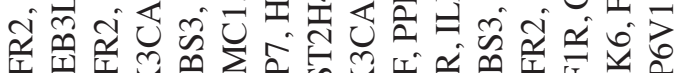

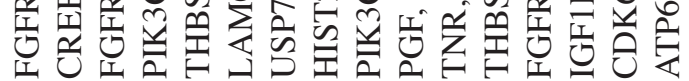

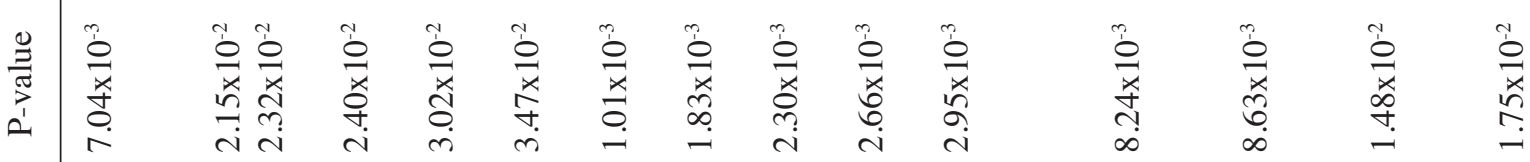

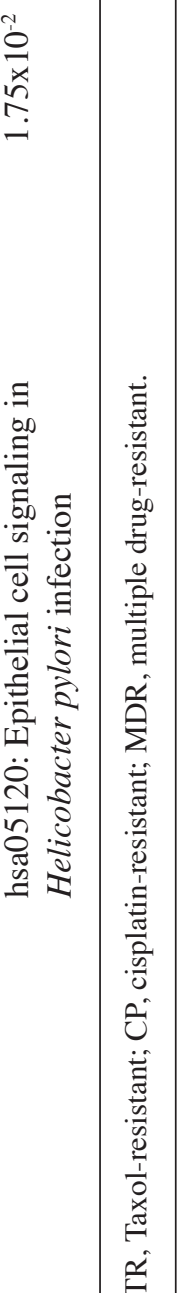




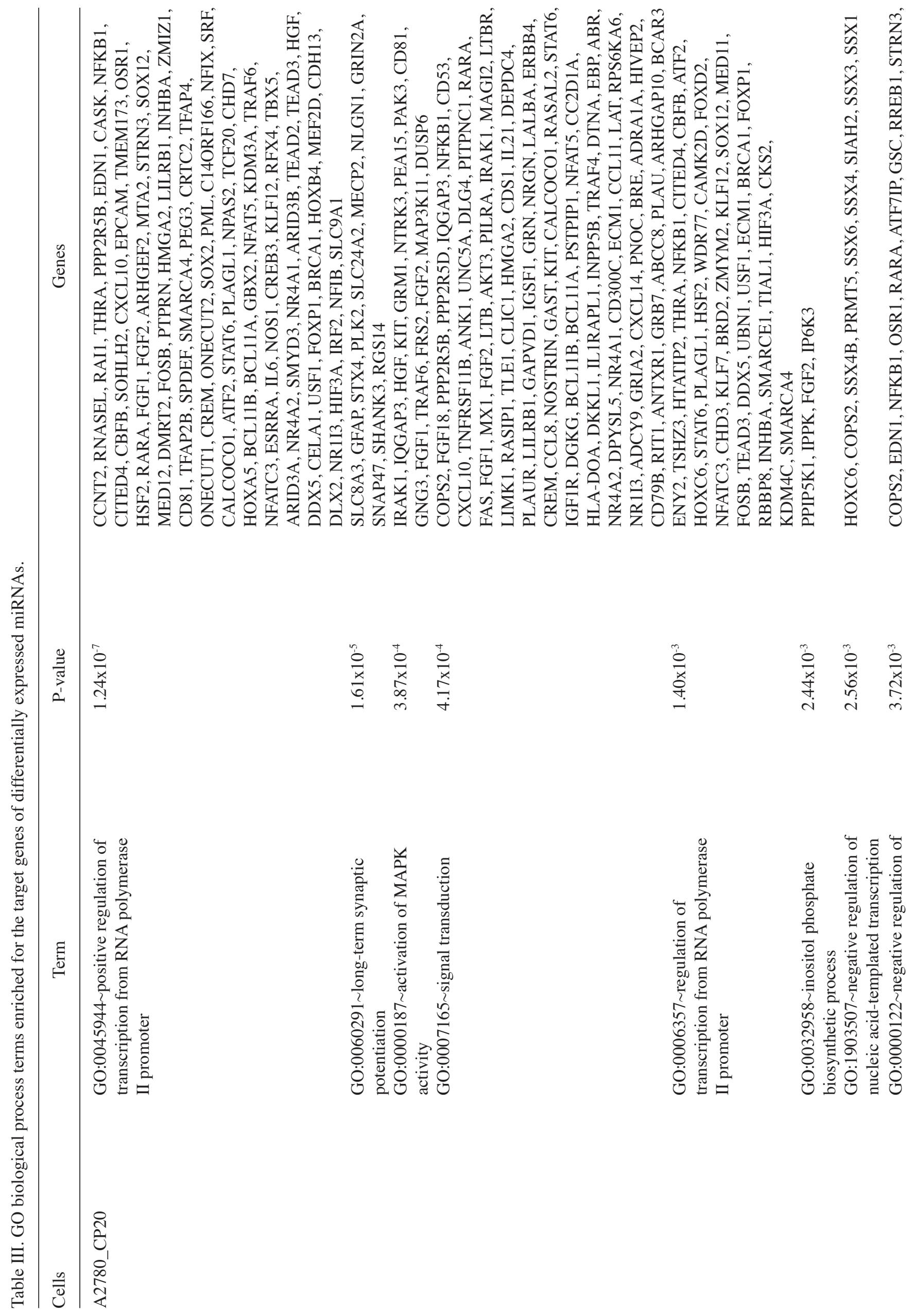




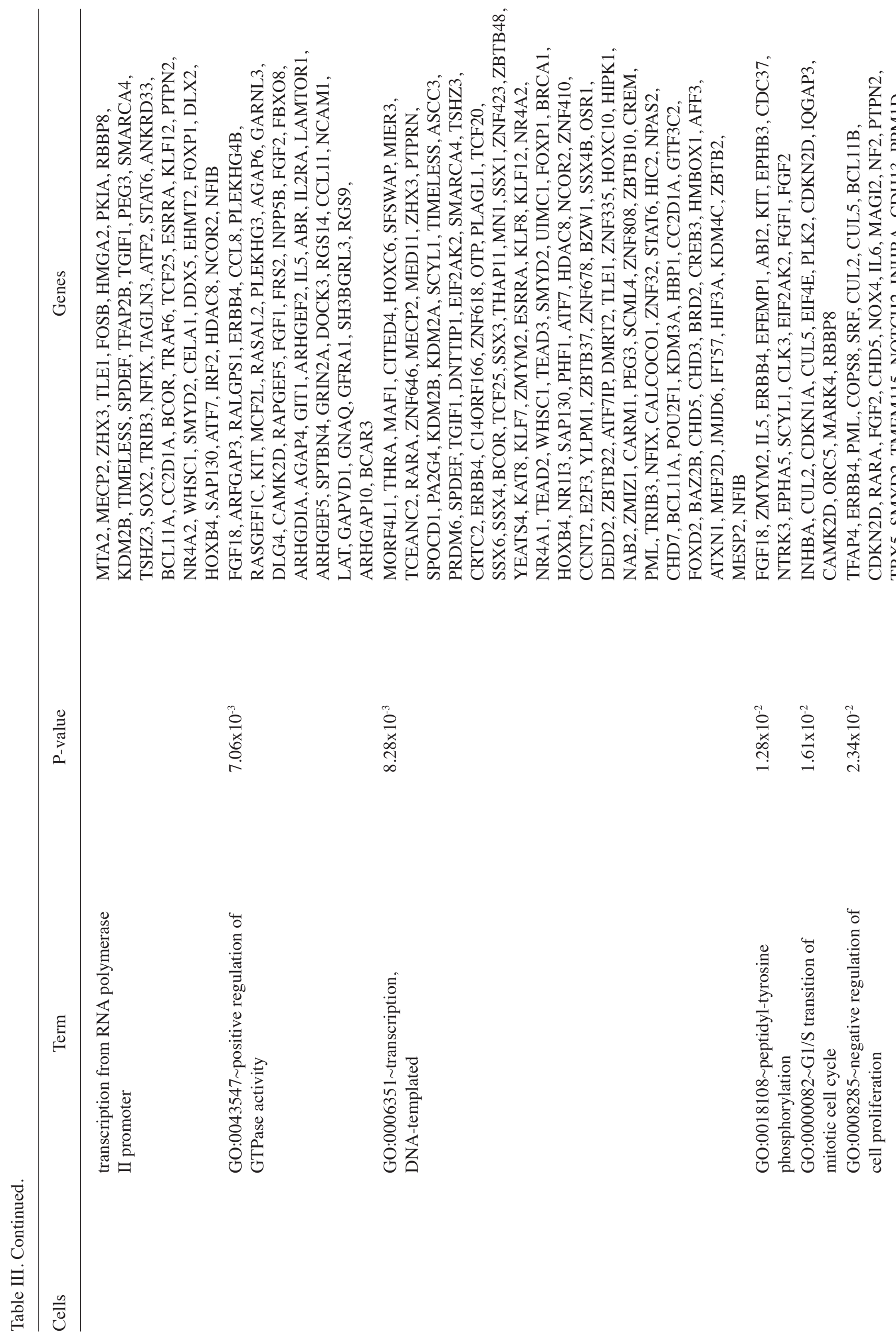




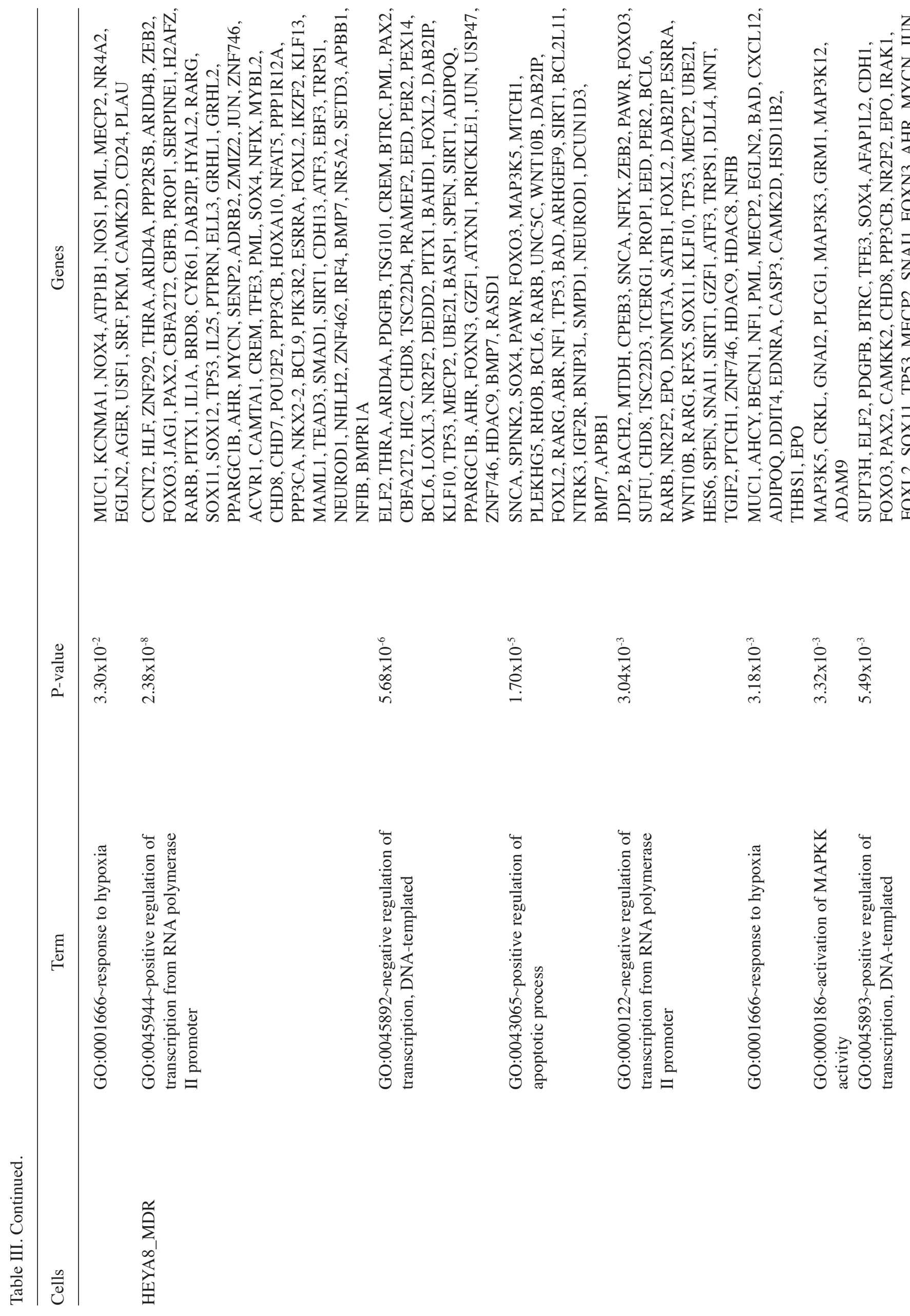




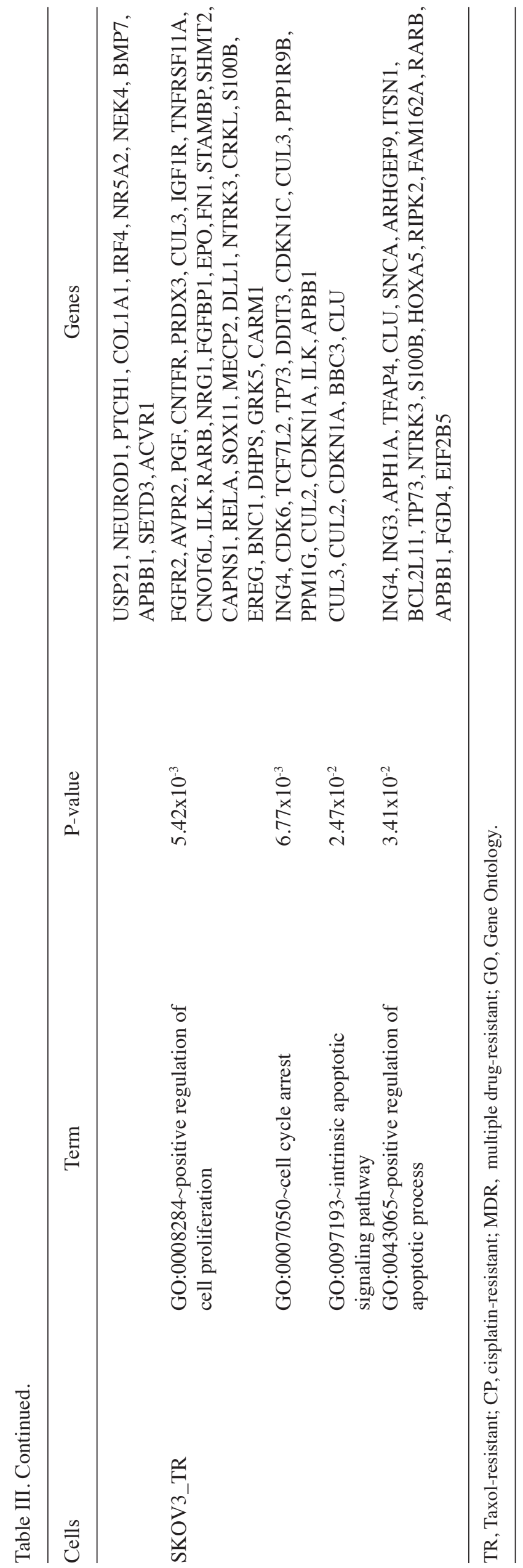




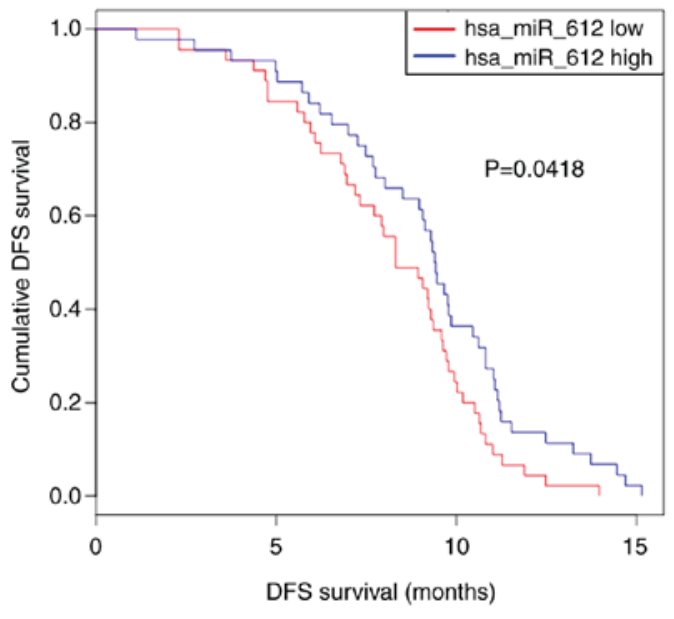

Figure 6. Kaplan-Meier curve displaying the correlation between miR-612 and DFS. miR, microRNA; DFS, disease-free survival.

DE-miRNAs may be involved in SKOV3_TR cell resistance by participating in the positive regulation of cell proliferation (miR-922-NTRK3 and miR-922-MECP2) and cell cycle arrest (miR-922-CUL2). According to these results, MECP2 and CUL2 were the commonly enriched target genes in the three cell lines. Since MECP2 is one of the target genes of hsa-miR-183-5p, it may be involved in chemoresistance by regulating the proliferation process.

TCGA validation. To validate the clinical importance of the identified miRNAs, the miRNA expression and survival data associated with OC were also extracted from the TCGA database. Kaplan-Meier analysis indicated that only the expression of miR-612 was significantly associated the DFS, with high expression of this miRNAs predicting poor patient prognosis (Fig. 6). No other miRNAs were observed to be associated with DFS or OS (data not shown).

\section{Discussion}

In the present study, common DE-miRNAs between resistant $\mathrm{OC}$ cells and exosomes were screened for the first time in A2780_CP20 (including miR-146b-5p, miR-509-5p, miR-574-3p, miR-574-5p and miR-760), HEYA8_MDR (including miR-30a-3p, miR-30a-5p, miR-612 and miR-617) and SKOV3_TR (including miR-193a-5p, miR-423-3p, miR-769-5p and miR-922). Furthermore, miR-574-3p, miR-30a-5p and miR-922 were suggested to participate in the drug resistance in OC by regulating the expression of CUL2 to mediate the HIF-1 cancer signaling pathway, cell proliferation and cell cycle arrest. Similar to miR-760, miR-30a-5p and miR-922, the shared hsa-miR-183-5p in the three cell lines may also be involved in drug-resistance by modulating MECP 2 and then influence the biological processes of response to hypoxia and cell proliferation. In addition, miR-612 was revealed to regulate TEAD3, serving important roles in multiple drug resistance via Hippo signaling pathway-mediated cell proliferation, and was significantly associated with prognosis. Accordingly, these cell proliferation-associated miRNAs may represent important targets for improving the chemotherapy sensitivity of OC.
HIF-1 $\alpha$ is a transcription factor that mediates the expression of hypoxia-sensitive genes, such as vascular endothelial growth factor, glucose transporter-1 and lactate dehydrogenase, which in turn promote angiogenesis and rapid tumor growth, thus inducing chemotherapy resistance $(19,20)$. Therefore, the inhibition or knockdown of HIF-1 $\alpha$ has been considered as an effective strategy to overcome the drug resistance in a number of diseases, including in OC $(21,22)$. CUL2 is a member of the cullin family of ubiquitin ligases, which associate with the von Hippel-Lindau tumor suppressor protein (VHL), the transcriptional elongation factors elongin B/C, RING-box protein RBX1 and E3 ubiquitin-protein ligase in order to recognize, ubiquitinate and ultimately induce the degradation of HIF- $\alpha(23,24)$. Thus, CUL2 may be downregulated in drug resistance cells, and its upstream miRNAs may display proto-oncogenic activity. This hypothesis has indirectly been demonstrated by a number of studies; for instance, Ghosh et al (25) identified that hypoxia induced the increased expression of miR-424, which then targeted CUL2 to stabilize HIF- $\alpha$ isoforms and promoted angiogenesis. miR- 424 was also reported to be upregulated in OC stem cells, chemoresistant OC cells and tumor tissue samples $(26,27)$. In line with these studies, the current study also identified three proto-oncogenic miRNAs, including miR-574-3p, miR-30a-5p and miR-922, in drug-resistant $\mathrm{OC}$ cells and exosomes. Although these three miRNAs were not previously demonstrated to be involved in $\mathrm{OC}$, their roles in other types of cancer indirectly explain these results. For instance, the relative expression of miR-574-3p was reported to be significantly higher in patients with hepatocellular carcinoma as compared with that in the cirrhosis patients and healthy controls (28). miR-30a-5p levels were also significantly increased in the serum and fine needle aspiration biopsy samples of malignant papillary thyroid carcinoma groups when compared with those in benign and atypical cells of undetermined significance (ACUS) (29).

MECP2 is a member of the methyl CpG binding proteins, which is capable of repressing transcription of a serials of genes, including brain derived neurotrophic factor (BDNF) $(30,31)$ and MDR1 (32), by specifically binding to methylated CpG dinucleotides and a transcriptional repression domain by interacting with histone deacetylase. Evidence demonstrated that $\operatorname{BDNF}(33,34)$ and MDR1 $(35)$ were significantly highly expressed in cancer and drug-resistant cells; thus, MECP2 may be downregulated in cancer. This was reported by the study of Zhu et al (36), which demonstrated that MECP2 protein levels in gastric cancer MDR cell lines SGC7901/VCR and SGC7901/ADR were significantly reduced as compared with those in parental SGC7901 cells (36). Therefore, the regulation of MECP2, such as by targeting its upstream miRNAs, may be a potential approach for the improvement of OC treatment. This hypothesis has been clarified in the study of Zhu et al (36), in which miR-19a/b was found to be significantly increased in SGC7901 cells subjected to 5-Aza-dC treatment to induce MDR, while pretreatment with miR19a/b inhibitors increased the expression of MECP2 in SGC7901 cells and prevented the MDR triggered by 5-Aza-dC. In the present study, four miRNAs (namely miR-183-5p, miR-760, miR-30a-5p and miR-922) that regulated the expression of MECP2 in OC drug-resistant cells were identified. As expected, miR-183-5p and miR-760 have been proven to serve 
as proto-oncogenes in cancer. For instance, Gao et al (37) revealed that the expression level of miR-183-5p was notably increased in bladder cancer tissues compared with adjacent non-cancerous tissues, and was markedly associated with papillary subtype and early pathological stage (I-II) according to the tumor-node-metastasis classification, with a diagnostic performance of $94.8 \%$ reported following the receiver operating characteristic analysis. Furthermore, Cheng et al (38) demonstrated that overexpression of miR-183-5p significantly enhanced the cell proliferation and inhibited cell apoptosis in the breast cancer cell lines MCF-7 and MDA-MB-231. The findings of Liao et al (39) also suggested that the expression of miR-760 was markedly upregulated in OC cell lines and tissues, and high miR-760 expression was associated with an aggressive phenotype and poor prognosis of OC patients. The proliferation of OC cells in vitro was reportedly promoted by the upregulation of miR-760 and inhibited by downregulation of miR-760 (39).

TEAD3, also known as TEF-5, is a member of the transcriptional enhancer factor family of transcription factors that promotes the transcription of a number of cell proliferation-associated genes (such as cyclins and cyclin-dependent kinases) and leads to cell cycle progression, contributing to the development of cancer and drug resistance $(40,41)$. It is, thus, speculated that its upstream miRNAs may be tumor suppressor genes. As anticipated, miR-612 was found to be downregulated in HEYA8_MDR cells and exosomes in the present study, and was associated with poor prognosis. The current results appear to be in line with previous studies, in which overexpression of miR-612 was reported to suppress the growth and metastasis of hepatocellular carcinoma and colorectal cancer, and to relieve drug resistance to cisplatin and 5-fluorouracil $(42,43)$.

However, a number of limitations exist in the current study that should be acknowledged when interpreting the results. Firstly, only three resistant OC cell lines induced by two chemotherapy drugs were selected for screening crucial miRNAs. Therefore, the miRNAs identified herein may not represent miRNA candidates that are potentially correlated with all types of OC and resistance to all drugs. Secondly, only two repeats were included in each sample of the microarray data used (13), which may influence the statistical results and lead to different results when using different cells. In addition, a normal control was not included in the TCGA data, which led to several unexpected, non-significant associations between our identified miRNAs in OC cells and the survival of patients. Thus, further clinical trials are required to further confirm the study conclusions. Furthermore, although the current analysis has preliminarily speculated the negative correlation between miRNAs and their target genes, in vitro and in vivo experiments are required. Finally, the exosomal mechanisms of the identified miRNAs remain uninvestigated in $\mathrm{OC}$.

In conclusion, the preliminarily results reported in the present study revealed that miR-574-3p, miR-30a-5p, hsa-miR-183-5p, miR-760 and miR-162 may be crucial for the development of drug-resistance in $\mathrm{OC}$ in an exosomal and non-exosomal manner. These miRNAs may be involved in OC by targeted modulation of the cell proliferation-associated genes CUL2, MECP2 and TEAD3. Upregulation or downregulation of these genes may be a potential therapeutic strategy for improving the drug sensitivity in OC. However, further studies are necessary to confirm these conclusions.

\section{Acknowledgements}

Not applicable.

\section{Funding}

Not applicable.

\section{Availability of data and materials}

The microarray data GSE76449 were downloaded from the GEO database in NCBI (http://www.ncbi.nlm.nih.gov/geo/).

\section{Authors' contributions}

YF, XX and QH conceived and designed the original study. YF and WH conducted the statistical analysis and drafted the manuscript. ZS, SDL, WX, YM and WB contributed to the acquisition and interpretation of data. XX and QH participated in critical revision of the manuscript. All authors read and approved the final manuscript.

\section{Ethics approval and consent to participate}

As the data used in this study were downloaded from the GEO database and no human experiments were involved in the study, obtaining informed consents was not required.

\section{Patient consent for publication}

Not applicable.

\section{Competing interests}

The authors declare that they have no competing interests.

\section{References}

1. Chen W, Zheng R, Baade PD, Zhang S, Zeng H, Bray F, Jemal A Yu XQ and He J: Cancer statistics in China, 2015. CA Cancer J Clin 66: 115-132, 2016.

2. Kosary CL: FIGO stage, histology, histologic grade, age and race as prognostic factors in determining survival for cancers of the female gynecological system: an analysis of 1973-87 SEER cases of cancers of the endometrium, cervix, ovary, vulva, and vagina. Semin Surg Oncol 10: 31-46, 1994.

3. Cliby WA, Powell MA, Alhammadi N, Chen L, Philip Miller J, Roland PY, Mutch DG and Bristow RE: Ovarian cancer in the United States: Contemporary patterns of care associated with improved survival. Gynecol Oncol 136: 11-17, 2015.

4. Hausser J and Zavolan M: Identification and consequences of miRNA-target interactions - beyond repression of gene expression. Nat Rev Genet 15: 599-612, 2014.

5. Li N, Yang L, Wang H, Yi T, Jia X, Chen C and Xu P: miR-130a and miR-374a function as novel regulators of cisplatin resistance in human ovarian cancer A2780 cells. PLoS One 10: e0128886, 2015.

6. Zou J, Liu L, Wang Q, Yin F, Yang Z, Zhang W and Li L: Downregulation of miR-429 contributes to the development of drug resistance in epithelial ovarian cancer by targeting ZEB1. Am J Transl Res 9: 1357-1368, 2017. 
7. Zhu H, Yang SY, Wang J, Wang L and Han SY: Evidence for miR-17-92 and miR-134 gene cluster regulation of ovarian cancer drug resistance. Eur Rev Med Pharmacol Sci 20: 2526-2531, 2016.

8. Kim TH, Jeong JY, Park JY, Kim SW, Jin HH, Kang H, Kim G and An HJ: miR-150 enhances apoptotic and anti-tumor effects of paclitaxel in paclitaxel-resistant ovarian cancer cells by targeting Notch3. Oncotarget 8: 72788-72800, 2017.

9. Yu DD, Wu Y, Zhang XH, Lv MM, Chen WX, Chen X, Yang SJ, Shen H, Zhong SL, Tang JH and Zhao JH: Exosomes from Adriamycin-resistant breast cancer cells transmit drug resistance partly by delivering miR-222. Tumour Biol 37: 3227-3235, 2016.

10. Qin X, Yu S, Zhou L, Shi M, Hu Y, Xu X, Shen B, Liu S, Yan D and Feng J: Cisplatin-resistant lung cancer cell-derived exosomes increase cisplatin resistance of recipient cells in exosomal miR-100-5p-dependent manner. Int J Nanomedicine 12 : 3721-3733, 2017.

11. Wei Y, Lai X, Yu S, Chen S, Ma Y, Zhang Y, Li H, Zhu X, Yao L and Zhang J: Exosomal miR-221/222 enhances tamoxifen resistance in recipient ER-positive breast cancer cells. Breast Cancer Res Treat 147: 423-431, 2014

12. Kanlikilicer P, Rashed MH, Bayraktar R, Mitra R, Ivan C, Aslan B, Zhang X, Filant J, Silva AM, Rodriguez-Aguayo C, et al: Ubiquitous release of exosomal tumor suppressor miR-6126 from ovarian cancer cells. Cancer Res 76: 7194-7207, 2016.

13. Rashed MH, Kanlikilicer P, Rodriguezaguayo C, Pichler M, Bayraktar R, Bayraktar E, Ivan C, Filant J, Silva A, Aslan B, et al: Exosomal miR-940 maintains SRC-mediated oncogenic activity in cancer cells: A possible role for exosomal disposal of tumor suppressor miRNAs. Oncotarget 8: 20145-20164, 2017.

14. Smyth GK: Limma: Linear models for microarray data. In Bioinformatics and computational biology solutions using $\mathrm{R}$ and bioconductor. Gentleman R, Carey VJ, Huber W, Irizarry RA and Dudoit S (eds). Springer, New York, NY, pp397-420, 2005.

15. Dweep H and Gretz N: miRWalk2.0: A comprehensive atlas of microRNA-target interactions. Nat Methods 12: 697, 2015.

16. Kohl M, Wiese S and Warscheid B: Cytoscape: Software for visualization and analysis of biological networks. Methods Mol Biol 696: 291-303, 2011

17. Vasaikar SV, Straub P, Wang J and Zhang B: LinkedOmics: Analyzing multi-omics data within and across 32 cancer types. Nucleic Acids Res 6: D956-D963, 2018.

18. Huang DW, Sherman BT and Lempicki RA: Systematic and integrative analysis of large gene lists using DAVID bioinformatics resources. Nat Protoc 4: 44-57, 2009.

19. Sun X, Vale M, Jiang X, Gupta R and Krissansen GW: Antisense HIF-1alpha prevents acquired tumor resistance to angiostatin gene therapy. Cancer Gene Ther 17: 532-540, 2010.

20. Tozzi F, Zhou Y, Chen J, Bose D, Fan F, Wang J, Brusher H, Widger W, Weihua Z and Ellis LM: Evaluation of glycolytic activity and HIF-1 $\alpha$ expression in chemoresistant colorectal cancer cells. J Clin Oncol 29 (Suppl): S415, 2011.

21. Su W, Huang L, Ao Q, Zhang Q, Tian X, Fang Y and Lu Y: Noscapine sensitizes chemoresistant ovarian cancer cells to cisplatin through inhibition of HIF-1 $\alpha$. Cancer Lett 305: 94-99, 2011.

22. Ai Z, Yang L, Qiu S and Fan Z: Overcoming cisplatin resistance of ovarian cancer cells by targeting HIF-1-regulated cancer metabolism. Cancer Lett 373: 36-44, 2016.

23. Tan M, Gu Q, He H, Pamarthy D, Semenza GL and Sun Y: SAG/ROC2/RBX2 is a HIF-1 target gene that promotes HIF-1 alpha ubiquitination and degradation. Oncogene 27: 1404-1411, 2008

24. Maeda Y, Suzuki T, Pan X, Chen G, Pan S, Bartman T and Whitsett JA: CUL2 is required for the activity of hypoxia-inducible factor and vasculogenesis. J Biol Chem 283: 16084-16092, 2008.

25. Ghosh G, Subramanian IV, Adhikari N, Zhang X, Joshi HP, Basi D, Chandrashekhar YS, Hall JL, Roy S, Zeng Y and Ramakrishnan S: Hypoxia-induced microRNA-424 targets CUL2 to stabilize HIF- $\alpha$ isoforms and promotes angiogenesis. J Clin Invest 120: 4141-4154, 2010.

26. Park YT, Jeong JY, Lee MJ, Kim KI, Kim TH, Kwon YD, Lee C, Kim OJ and An HJ: MicroRNAs overexpressed in ovarian ALDH1-positive cells are associated with chemoresistance. J Ovarian Res 6: 18, 2013.
27. Ji HY, Lim J, Ha IS, Ji MS, Kim JH, Kim J, Chu WN and Cho YS: MicroRNA sequencing detects miR-424-5p up-regulation in ovarian cancer stem cells. Genes Genomics 37 737-742, 2015

28. Shen X, Xue Y, Cong H, Wang X and Ju S: Dysregulation of serum microRNA-574-3p and its clinical significance in hepatocellular carcinoma. Ann Clin Biochem 55: 478-484, 2018

29. Igci YZ, Ozkaya M, Korkmaz H, Bozgeyik E, Bayraktar R, Ulasli M, Erkilic S, Eraydin A and Oztuzcu S: Expression levels of miR-30a-5p in papillary thyroid carcinoma: A comparison between serum and fine needle aspiration biopsy samples. Genet Test Mol Biomarkers 19: 418-423, 2015.

30. Chen WG, Chang Q, Lin Y, Meissner A, West AE, Griffith EC, Jaenisch R and Greenberg ME: Derepression of BDNF transcription involves calcium-dependent phosphorylation of MeCP2 Science 302: 885-889, 2003.

31. Wade PA: Dynamic regulation of DNA methylation coupled transcriptional repression: $\mathrm{BDNF}$ regulation by $\mathrm{MeCP} 2$. Bioessays 26: 217-220, 2004

32. Elosta A, Kantharidis P, Zalcberg JR and Wolffe AP: Precipitous release of methyl-CpG binding protein 2 and histone deacetylase 1 from the methylated human multidrug resistance gene (MDR1) on activation. Mol Cell Biol 22: 1844-1857, 2002.

33. Au CW, Siu MK, Liao X, Wong ES, Ngan HY, Tam KF, Chan DC, Chan QK and Cheung AN: Tyrosine kinase B receptor and BDNF expression in ovarian cancers-Effect on cell migration, angiogenesis and clinical outcome. Cancer Lett 281: 151-161, 2009.

34. Lee J, Jiffar T and Kupferman ME: A novel role for BDNF-TrkB in the regulation of chemotherapy resistance in head and neck squamous cell carcinoma. PLoS One 7: e30246, 2012.

35. Li Z, Hu S, Wang J, Cai J, Xiao L, Yu L and Wang Z: miR-27a modulates MDR1/P-glycoprotein expression by targeting HIPK2 in human ovarian cancer cells. Gynecol Oncol 119: 125-130, 2010.

36. Zhu F, Wu Q, Ni Z, Lei C, Li T and Shi Y: miR-19a/b and MeCP2 repress reciprocally to regulate multidrug resistance in gastric cancer cells. Int J Mol Med 42: 228-236, 2018.

37. Gao JM, Huang LZ, Huang ZG and He RQ: Clinical value and potential pathways of miR-183-5p in bladder cancer: A study based on miRNA-seq data and bioinformatics analysis. Oncol Lett 15: 5056-5070, 2018.

38. Cheng Y, Xiang G, Meng Y and Dong R: miRNA-183-5p promotes cell proliferation and inhibits apoptosis in human breast cancer by targeting the PDCD4. Reprod Biol 16: 225-233, 2016.

39. Liao Y, Deng Y, Liu J, Ye Z, You Z, Yao S and He S: miR-760 overexpression promotes proliferation in ovarian cancer by downregulation of PHLPP2 expression. Gynecol Oncol 143: 655-663, 2016

40. Teng K, Deng C, Xu J, Men Q, Lei T, Di D, Liu T, Li W and Liu X: Nuclear localization of TEF3-1 promotes cell cycle progression and angiogenesis in cancer. Oncotarget 7: 13827-13841, 2016.

41. Qiao Y, Lin SJ, Chen Y, Voon DC, Zhu F, Chuang LS, Wang T, Tan P, Lee SC, Yeoh KG, et al: RUNX3 is a novel negative regulator of oncogenic TEAD-YAP complex in gastric cancer. Oncogene 35: 2664-2674, 2016.

42. Tang J, Tao ZH, Wen D, Wan JL, Liu DL, Zhang S, Cui JF, Sun HC, Wang L, Zhou J, et al: miR-612 suppresses the stemness of liver cancer via Wnt/ $\beta$-catenin signaling. Biochem Biophys Res Commun 447: 210-215, 2014.

43. Sheng L, He P, Yang X, Zhou M and Feng Q: miR-612 negatively regulates colorectal cancer growth and metastasis by targeting AKT2. Cell Death Dis 6: e1808, 2015.

This work is licensed under a Creative Commons Attribution-NonCommercial-NoDerivatives 4.0 International (CC BY-NC-ND 4.0) License. 